\title{
NOUVELL
}

\section{Vers une thérapie cellulaire des pathologies rétiniennes}

Joël Crouzet, Martin Neuner-Jehle, Pierre-Olivier Couraud
$>$ La proportion de sujets atteints de dégénérescence maculaire liée à l'âge dépasse $6 \%$ des personnes âgées de plus de 65 ans [1]. Cette pathologie rétinienne conduit à la perte de la vision centrale, affecte en partie les cellules de l'épithélium pigmentaire de la rétine et la membrane de Bruch au niveau de la macula, la partie centrale de la rétine (Figure 1). Les cellules de l'épithélium pigmentaire de la rétine sont indispensables à la vision. En effet, elles sont au contact direct des cellules photoréceptrices, responsables de la phototransduction. Ce contact leur permet de recycler les photopigments des cellules photoréceptrices et de participer au renouvellement des segments externes de ces cellules par phagocytose (Figure 1).

\section{Transplantation de cellules de} l'épithélium pigmentaire de la rétine dans la dégénérescence maculaire La perte des cellules de l'épithélium pigmentaire de la rétine ou de certaines de leurs fonctions semble jouer un rôle prépondérant dans la progression des diverses formes cliniques de la dégénérescence maculaire liée à l'âge qui sont associées soit à une néovascularisation choroïdienne envahissant la rétine (formes dites humides), soit à une atrophie de la choroïde et de la neurorétine (formes dites sèches). Cette observation a conduit à envisager comme approche thérapeutique le remplacement d'une partie des cellules de l'épithélium pigmentaire de la rétine. De fait, des translocations de neurorétine, d'une zone lésée vers une zone saine, possédant des cellules de l'épithélium pigmentaire de la rétine fonctionnelles, ont permis de blo-
J. Crouzet,

M. Neuner-Jehle :

Neurotech SA, Bâtiment

Génopole-Industries, 4 , rue Pierre Fontaine, 91000 Évry, France.

P.O. Couraud: Institut Cochin, Département de Biologie Cellulaire, 22, rue Méchain, 75014 Paris, France.
Deux lignées humaines de cellules de l'épithélium pigmentaire, dont l'absence de potentiel tumoral a été vérifiée chez la souris nude, ont été utilisées dans cette étude. Une de ces lignées est immortalisée de manière spontanée, et le gène $T$ de SV40 a été introduit dans l'autre. Ces cellules ont conservé les propriétés de cellules de l'épithélium pigmentaire : expression de marqueurs quer la progression de la maladie chez certains patients, voire d'améliorer leur acuité visuelle, montrant que la neurorétine peut être préservée fonctionnellement si on remplace la monocouche de CEP avec laquelle elle est en contact [2] .

Des transplantations de cellules de l'épithélium pigmentaire de rétine humaine fœtale ont déjà été réalisées chez l'homme [3] montrant la faisabilité de cette approche et l'absence d'effets secondaires majeurs. Toutefois, plusieurs contraintes importantes limitent le développement d'une telle approche: (1) les cellules fœtales ne peuvent pas être obtenues en grande quantité, la survenue de la sénescence limitant leur multiplication in vitro; (2) la reproductibilité et la sécurité sanitaire de ces prélèvements sont difficiles à contrôler. L'utilisation de cellules de l'épithélium pigmentaire humaines immortalisées pourrait constituer une alternative thérapeutique intéressante. En effet, des lignées de cellules immortalisées présentent l'avantage de pouvoir être produites à grande échelle selon des procédures standardisées. C'est cette approche qui a été développée récemment dans le cadre d'une collaboration réunissant des équipes de l'Institut d'Ophtalmologie (University College de Londres, GB), de l'Université de Sheffield (GB) et de la société française Neurotech située à Évry (France) [4]. cellulaires spécifiques et capacité de phagocytose des segments externes des cellules.

L'efficacité thérapeutique de ces lignées a été testée après leur greffe chez des rats provenant d'une souche particulière, le rat RCS : une mutation génétique prive les cellules de l'épithélium pigmentaire de ces animaux de leur capacité de phagocytose au niveau des segments externes [5]. Ce défaut se traduit par une perte progressive des cellules photoréceptrices (dès l'âge de 3 semaines et jusqu'à 5 mois) entraînant une perte de la vision. Des cellules de l'épithélium pigmentaire ont été injectées dans l'espace sous-rétinien de ces rats (Figure 1), au contact des cellules de l'épithélium pigmentaire de l'hôte, au moment où la dégénérescence des cellules photoréceptrices apparaît. Cinq mois plus tard, l'analyse histologique chez les animaux greffés avec des cellules humaines de l'épithélium pigmentaire, montre que les cellules photoréceptrices persistent autour du site d'injection, ce qui n'est pas le cas chez les animaux témoins. Sur le plan fonctionnel, les résultats sont tout à fait intéressants. En effet, un test de comportement visuel a montré que les animaux greffés répondaient sensiblement mieux que les animaux non injectés ou injectés avec du tampon physiologique seul. Dix semaines après la greffe, 
la réponse observée était similaire à celle de rats normaux, exempts de l'anomalie des rats RCS. L'analyse électrophysiologique au niveau de l'aire visuelle cérébrale (le colliculus supérieur), en réponse à un stimulus visuel, a également démontré une sauvegarde des fonctions visuelles autour du site d'injection. Ce travail a donc permis de montrer que la greffe de lignées humaines de cellules de l'épithélium pigmentaire permet d'atténuer sensiblement la perte de fonctions visuelles en protégeant les cellules photoréceptrices au niveau du site d'injection. Il faut noter que les cellules immortalisées marquées au BrdU (bromodésoxy-uridine) puis greffées, ont été mises en évidence par immunohistochimie chez l'animal 5 mois après la greffe. Les cellules greffées sont retrouvées intégrées dans la monocouche de cellules de l'épithélium pigmentaire de l'hôte, au niveau de la zone de sauvegarde de cellules photoréceptrices. En outre ces cellules greffées présentent une polarisation de la mélanine similaire à celle des cellules de l'épithélium pigmentaire de I'hôte, reflétant un degré de différenciation élevé.

Ces résultats ouvrent des perspectives tout à fait intéressantes car des lignées humaines immortalisées de cellules de l'épithélium pigmentaire pourraient être utilisées à des fins de thérapie de remplacement, dans le cas de la dégénérescence maculaire liée à l'âge, pour pallier l'absence de cellules de l'épithélium pigmentaire ou leur dysfonctionnement métabolique à la suite de l'exérèse de membrane vasculaire dans les formes humides. Elles pourraient aussi s'avérer utiles dans certaines formes de rétinites pigmentaires et dans l'amaurose congénitale de Leber où le défaut géné- tique est associé à un déficit fonctionnel des cellules de l'épithélium pigmentaire [6].

\section{Autres approches de thérapie cellulaire dans les pathologies de l'œil \\ D'autres approches de thérapie} cellulaire sont actuellement en cours d'évaluation dans les pathologies de l'œil, notamment la rétinite pigmentaire. Ainsi, l'équipe de J. Sahel a mis en place une étude pilote chez l'homme pour évaluer l'effet de la greffe sous-rétinienne de feuillets de rétine humaine chez des patients atteints de rétinite pigmentaire. Cette maladie se traduit par une dystrophie progressive des cellules photoréceptrices. Les feuillets implantés libèrent un facteur de protection des cônes, la sous-population de cellules photoréceptrices se situant essentiellement au niveau de la macula [7]. Citons également dans cette maladie l'utilisation de cellules humaines produisant un facteur neuroprotecteur, et encapsulées dans des dispositifs médicaux de porosité contrô- lée. Les cellules sont ainsi isolées sur le plan immunologique évitant tout rejet. Après implantation dans l'humeur aqueuse de la chambre oculaire postérieure, il y a une libération continue par la capsule du principe actif qui peut atteindre la rétine par diffusion et conduire à la préservation des cellules photoréceptrices. Une preuve d'efficacité a été obtenue dans un modèle canin de rétinite pigmentaire [8]. L'intérêt de cette méthode réside essentiellement dans le fait qu'elle permet de délivrer un principe actif au niveau de la rétine, après une chirurgie simple, alors que la barrière hémato-rétinienne rend la rétine difficilement accessible par voie sanguine. Cette approche pourrait être
1. Klein R, Klein BEK, Linton KLP. Prevalence of agerelated maculopathy: the Beaver Dam Eye Study. Ophthalmology 1992 ; 99: 933-45.

2. De Juan $\varepsilon$, Loewenstein A, Bressler NM, Alexander J. Translocation of the retina for management of subfoveal choroidal neovascularization II: a preliminary report in humans. Am J Ophthalmol 1998 ; 125: 698-700.

3. Algvere PV, Berglin L, Gouras P, Sheng Y. Transplantation of fetal retinal pigment epithelium in age-related macular degeneration with subfoveal neovascularization. Graefes Arch Clin Exp Ophthalmol $1994 ; 232$ : 707-16.

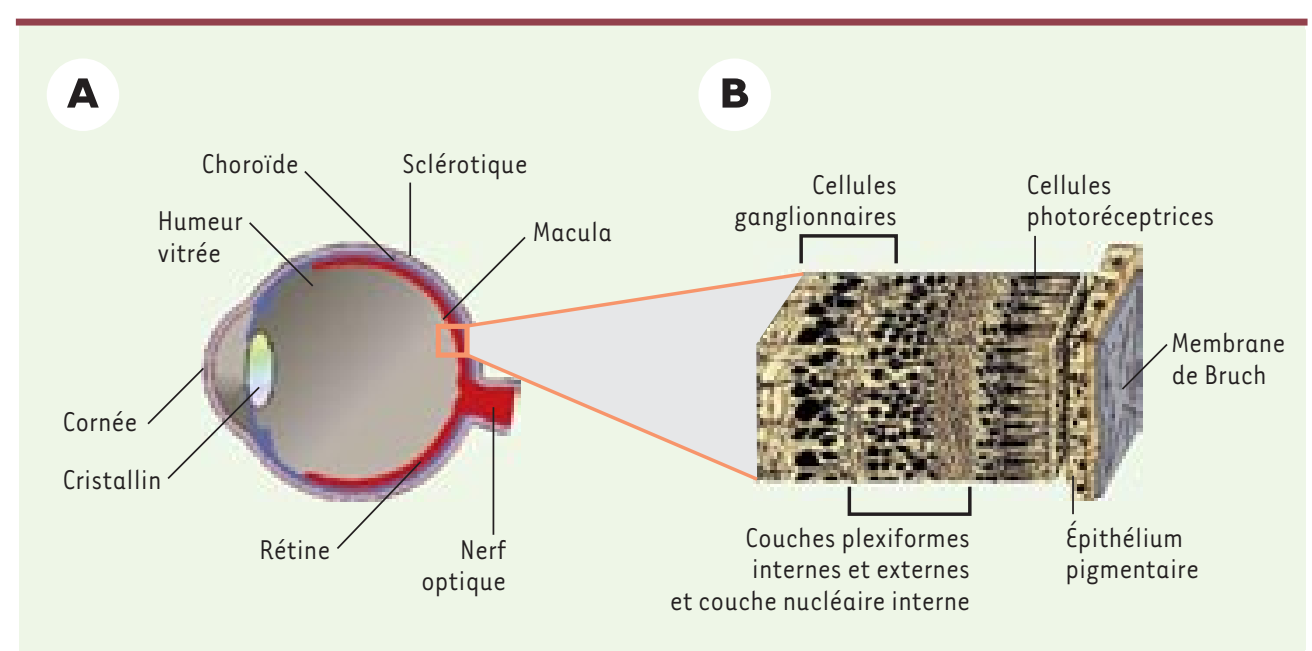

Figure 1. Anatomie de la rétine. A. Coupe sagittale de l'œil humain et agrandissement de la rétine montrant l'organisation cellulaire de celle-ci $\boldsymbol{B}$. Les cellules photoréceptrices sont en contact direct avec l'épithélium pigmentaire de la rétine, reposant lui-même sur la membrane de Bruch. Le réseau capillaire de la choroïde, enveloppant la face externe de la membrane de Bruch, est responsable des apports nutritifs et de l'oxygénation de la rétine externe. 
appliquée à d'autres maladies de la rétine. Les approches de thérapie cellulaire pour les pathologies rétiniennes laissent donc entrevoir des perspectives thérapeutiques intéressantes. $\Delta$

Cell therapy for retinal diseases
4. Lund RD, Adamson P, Sauve $Y$, et al. Subretinal transplantation of genetically modified human cell lines attenuates loss of visual function in dystrophic rats. Proc Nat Acad Sci USA 2001 ; 98 9942-7.

5. Glodman Al, O'Brien PJ. Phagocytosis in the retinal pigment epithelium of the RCS rat. Science 1978 ; $201: 1023-5$

\section{NOUVELLE}

\section{Les ARN modulent la transcription}

Olivier Bensaude, Annemieke A. Michels, Van Trung Nguyen
6. Morimura $\mathrm{H}$, Fishman $\mathrm{GA}$, Grover SA, Fulton $A B$, Berson $\varepsilon L$, Dryja TP. Mutations in the RPE65 gene in patients with autosomal recessive retinitis pigmentosa or Leber congenital amaurosis. Proc Natl Acad Sci USA 1998 ; 95 : 3088-93.

7. Mohand-Said S, DeudonCombe A, Hicks D, et al. Normal retina releases a diffusible factor stimulating cone survival in the retinal degeneration mouse. Proc Natl Acad Sci USA 1998 ; 95 : 8357-62.

8. Pierce-Kelling $S E$, Rein D, Acland GM, et al. Encapsulated cell-based intraocular delivery of CNTF slows inherited retinal degeneration in the rcdl dog model. Invest Ophthalmol Vis Sci 2000 ; 41 : S542. mouche pour stimuler l'acétylation des histones. En revanche, le mode d'action de Xist chez la souris n'est pas connu,

Cnrs UMR 8541,
Régulation
de l'Expression Génétique,
École Normale Supérieure,
46 , rue d'Ulm,
75230 Paris Cedex 05,
France.
mais il est lui-même réglé négativement $(\rightarrow) \mathrm{m} / \mathrm{s}$ par un transcrit 2000, $\mathbf{n}^{\circ} \mathbf{6 - 7}$, antisens, Tsix $(\rightarrow)$.

Des ARN modulent directement l'activité de facteurs de transcription spécifiques. Ainsi, dans l'œuf de xénope, l'association avec un (des) ARN maintiendrait dans le cytoplasme le facteur de transcription CBTF (CCAAT-box transcription factor) [5] et l'association avec des ARN messagers polyadénylés supprime la capacité de liaison à I'ADN du facteur de transcription Yin Yang l (yyl) [6]. En utilisant un crible double-hybride, l'équipe de Bert O'Malley (Houston, TX, USA), a découvert de façon inattendue un ARN, le SRA (steroïd receptor RNA activator), co-activateur de la transcription des gènes activés par les récepteurs des stéroïdes [7] $(\rightarrow)$. Cet ARN polyadénylé présente $(\rightarrow) \mathrm{m} / \mathrm{s}$ de courtes phases de lecture 1999, ouverte, mais la destruction $n^{\circ} 10$, p. 1153 initiateurs de ces phases n'a pas de conséquence fonctionnelle. L'ARN SRA peut s'associer à une protéine à «boîte $D E A D »^{*}$ du complexe co-activateur des récepteurs des stéroïdes [8]. Plus récem-

* Famille de protéines se liant aux ARN et dont la séquence comprend le motif d'acides aminés : -D-E-A-D-. 